\title{
SCAN: A novel approach for vancomycin time-out
}

\author{
Kayihura Manigaba PharmD ${ }^{1}$, Samuel J. Borgert PharmD ${ }^{1}$, Kenneth P. Klinker PharmD ${ }^{2}$, Kartikeya Cherabuddi MD ${ }^{3}$ \\ and Veena Venugopalan PharmD ${ }^{2}$ \\ ${ }^{1}$ University of Florida Health Shands Hospital, Gainesville, Florida and, ${ }^{2}$ College of Pharmacy, University of Florida, Gainesville, Florida and ${ }^{3}$ University of \\ Florida, College of Medicine, Gainesville, Florida
}

To the Editor-Approximately $30 \%-50 \%$ of antimicrobials prescribed in hospitals are either inappropriate or unnecessary. ${ }^{1}$ The Joint Commission (TJC), Centers for Disease Control (CDC), and Infectious Diseases Society of America (IDSA) in partnership with the Society of Healthcare Epidemiology (SHEA) have all issued performance elements for antimicrobial stewardship programs (ASP). ${ }^{2-4}$ One core element in these standards is use of a systematic process for evaluating the need for treatment after a set period of time, most often referred to as "antibiotic time-out." Although there is unanimous agreement that use of prospective strategies to evaluate antibiotic treatment is essential, little guidance on how to best integrate an antibiotic time-out process into clinical practice exists. We present an innovative approach to perform vancomycin time-out in a hospital setting.

\section{Methods}

The Stop and ReConsider Advancing CoNsult (SCAN) policy was implemented at our institution in November 2017 after approval from the hospital anti-infective subcommittee and the Pharmacy and Therapeutics Committee. In this policy, one of the first steps taken by the pharmacist is review of microbiological data to ensure that appropriate cultures are obtained (Fig. 1). Next, 72 hours from initiation of vancomycin, the SCAN protocol is triggered which requires the pharmacist to assess criteria for continuation of therapy. These criteria include positive cultures for methicillin-resistant Staphylococcus aureus (MRSA), history of MRSA, culture results $\leq 24-$ $48 \mathrm{~h}$, severe $\beta$-lactam allergy with no alternative antibiotics, and purulent cellulitis. If criteria are met, vancomycin therapy is continued. If criteria are not met, the pharmacist communicates with the primary team and either recommends discontinuing vancomycin or consultation with the infectious diseases service if continuation of therapy is needed. The SCAN protocol permits deviation from predefined criteria in cases of clinical instability or critical illness. A training video was developed by the ASP, discussing how SCAN would be integrated into pharmacist workflow. Prior to performing SCAN, pharmacists are required to take this training and to pass a competency assessment. Pharmacists are required to document SCAN using a standardized template in the electronic medical record (EMR).

We conducted a 3-month retrospective review following the implementation of SCAN to assess pharmacist adherence to this new process and impact on vancomycin utilization. Patients with

\footnotetext{
Author for correspondence: Veena Venugopalan, PharmD, BCPS, Department of Pharmacotherapy and Translational Research, University of Florida, College of Pharmacy, P.O Box 100484, Gainesville, FL 32610. Email: vvenugopalan@cop.ufl.edu

Cite this article: Manigaba K, et al. (2018). SCAN: A novel approach for vancomycin time-out. Infection Control \& Hospital Epidemiology 2018, 39, 1501-1502. doi: 10.1017/ ice.2018.249

a pharmacokinetic $(\mathrm{PK})$ consult, who received $\geq 48$ hours of vancomycin were included in the analysis. Patients who were followed by the infectious diseases consult service during the first 48 hours of vancomycin therapy were excluded. The University of Florida Institution Review Board approved this study as a quality improvement project.

\section{Results}

In total, 269 patients met inclusion criteria for analysis. Pharmacists performed SCAN at 72 hours in 157 of 269 (58\%) of patients, meaning they assessed presence of indications for vancomycin use. The most common reasons for continuation of vancomycin beyond 72 hours was pending culture (26 of 157 patients, $17 \%$ ), positive cultures or history of MRSA (26 of 157 patients, 17\%), and purulent cellulitis (7 of 157 patients, $4 \%)$. Vancomycin was discontinued in 89 of 157 patients of the SCAN group (57\%) compared to 32 of 112 patients in the nonSCAN group $(29 \% ; P<.05)$. The median length of vancomycin therapy was 3.3 days (range, 2.6-4.7 days) in the SCAN group compared to 4.1 days (range, 2.9-5.9 days) in the non-SCAN group $(P<.05)$. Prior to the implementation of SCAN, the average length of vancomycin therapy was 8 days based on an internal audit.

\section{Discussion}

Vancomycin monitoring through a PK consultation service is a pharmacist-lead initiative at most institutions. Historically, the focus of this consultative service has been on vancomycin dosing accuracy with less emphasis on treatment appropriateness. The SCAN protocol is unique because it incorporates an evaluation of vancomycin use at 72 hours into the PK consult. With any new process, we noticed a ramp-up phase for adoption of this protocol. In the first month, the compliance rate was approximately $50 \%$, and it increased to $65 \%$ in the third month postimplementation. There was a significant decline in vancomycin days of therapy in the SCAN group versus the non-SCAN group (3.3 vs 4.1 days; $P<.05$ ). The implementation of the SCAN protocol was motivated by the need to improve the vancomycin PK consult workflow, decrease antibiotic utilization, and integrate antibiotic stewardship strategies into existing institutional policies. The impact of SCAN potentially extends beyond vancomycin. Following implementation, front-line pharmacists are reporting enhanced stewardship discussions on all prescribed antibiotics, not just vancomycin. This is an intriguing finding and supports the role of active communication when integrating stewardship into practice. ${ }^{5,6}$ Based on feedback from pharmacists, one barrier to performing SCAN was fear of missing 


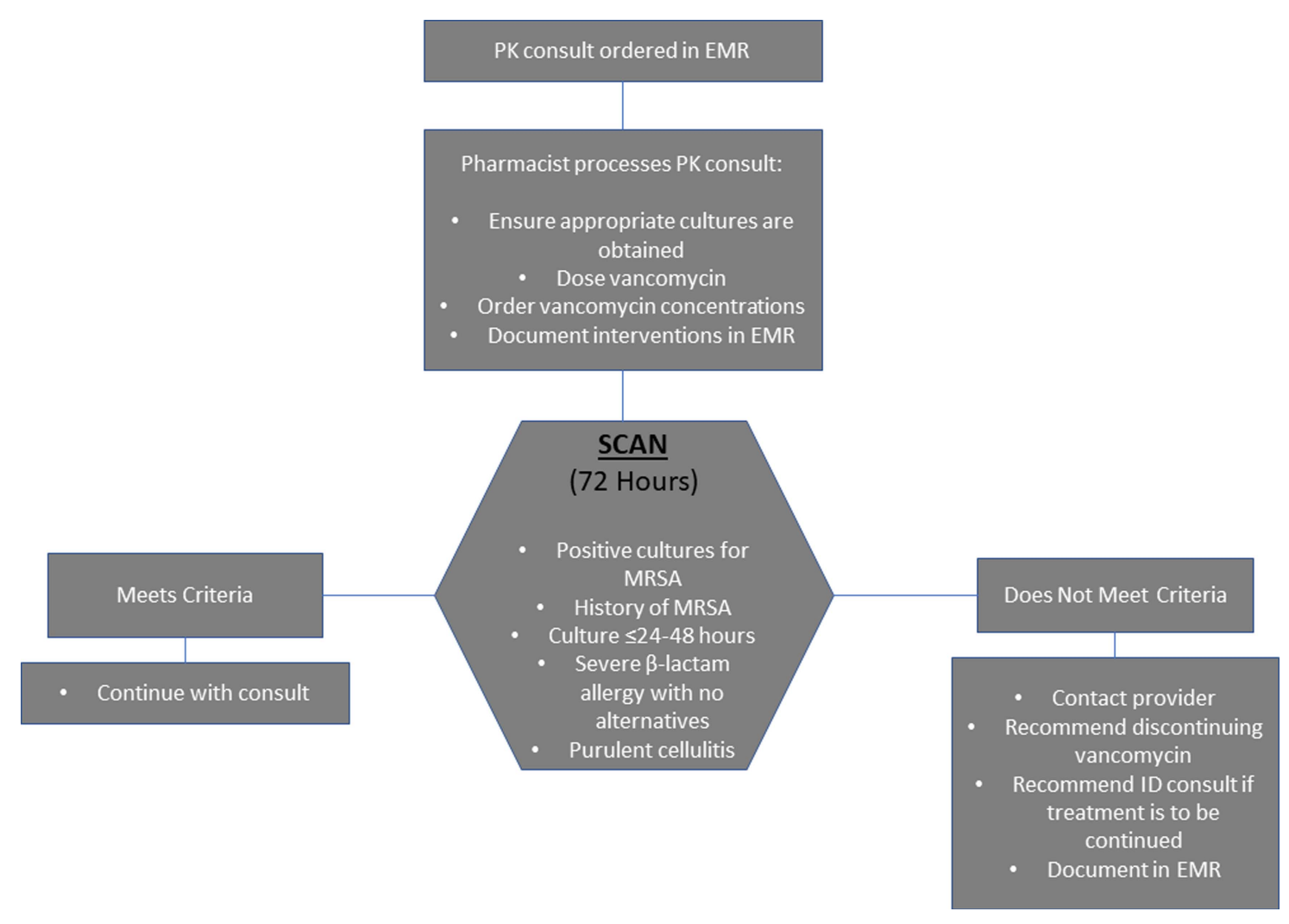

Figure. 1. Vancomycin PK Workflow

critical patient information or lack of in-depth knowledge of the patient's clinical condition.

Strategies for antibiotic time-out are centered on prompting prescribers to assess antibiotic needs or automatically stopping antibiotics to trigger review prior to renewal. ${ }^{7,8}$ Prior studies have demonstrated that use of electronic checklists integrated into the EMR in combination with face-to-face conversations with providers are effective in prompting antibiotic review and reducing antibiotic utilization. ${ }^{5,6}$ Although there are reports of the successful use of automatic vancomycin stop orders in reducing inappropriate use, one concern frequently raised by clinicians is potential interruptions in therapy in cases where continuation is desired. ${ }^{8,9}$ SCAN differs from an automatic stop order because the medication is not discontinued rather, a discussion regarding vancomycin use is initiated between the pharmacist and provider.

Hospitals with existing PK consult services could integrate a process similar to SCAN into their workflow to optimize vancomycin utilization. SCAN serves as a blueprint for an effective "antibiotic time-out" process particularly in settings with limited antimicrobial stewardship resources.

\section{Acknowledgments.}

Financial support. No financial support was provided relevant to this article.

Conflicts of interest. Dr. Borgert reports serving on advisory board for Theravance Pharmaceuticals. Dr. Klinker reports personal fees from Melinta Therapeutics, personal fees from Nabriva Therapeutics, and personal fees from Achaogen, all outside the submitted work. All other authors report no conflicts of interest relevant to this article.

\section{References}

1. CDC. Antibiotic prescribing and use in hospitals and long-term care. Centers for Disease Control and Prevention website. https://www.cdc.gov/ antibiotic-use/healthcare/. Updated February 2018. Accessed June 25, 2018.

2. O'Neill J. Review on antimicrobial resistance: tackling drug-resistant infections globally-final report and recommendations. Review on Antimicrobial Resistance website. https://amr-review.org/sites/default/ files/160518_Final\%20paper_with\%20cover.pdf. Published 2016. Accessed June 25, 2018.

3. Approved: new antimicrobial stewardship standard. Jt Comm Perspect 2016;36:3-4,8.

4. Barlam TF, Cosgrove SE, Abbo LM et al. Implementing an antibiotic stewardship program: guidelines by the Infectious Diseases Society of America and the Society for Healthcare Epidemiology of America. Clin Infect Dis 2016;15;62:e51-e77.

5. Lee TC, Frenette C, Jayaraman D, Green L, Pilote L. Antibiotic selfstewardship: trainee-led structured antibiotic time-outs to improve antimicrobial use. Ann Intern Med 2014;18:161(10 Suppl):S53-S58.

6. Weiss CH, Dibardino D, Rho J, Sung N, Collander B, Wunderink RG. A clinical trial comparing physician prompting with an unprompted automated electronic checklist to reduce empirical antibiotic utilization. Crit Care Med 2013;41:2563-2569.

7. Guglielmo B, Dudas V, Maewal I, et al. Impact of a series of interventions in vancomycin prescribing on use and prevalence of vancomycin-resistant Enterococci. Jt Comm Qual Patient Saf 2005;31:469-475.

8. Connor DM, Binkley S, Fishman NO, Gasink LB, Linkin D, Lautenbach E. Impact of automatic orders to discontinue vancomycin therapy on vancomycin use in an antimicrobial stewardship program. Infect Control Hosp Epidemiol 2007;28:1408-1410.

9. Graber CJ, Jones MM, Glassman PA, et al. Taking an antibiotic time-out: utilization and usability of a self-stewardship time-out program for renewal of vancomycin and piperacillin-tazobactam. Hosp Pharm 2015;50:1011-1024. 